Mémoire.

\title{
CHOROLOGIE DES PHLÉBOTOMES DE L'EST ALGÉRIEN. (DIPTERA, PHLEBOTOMIDAE)
}

\author{
J. RUSSO*, J.-A RIOUX**, M. LAMBERT ${ }^{* *}$, P. RISPAIL **, A. BELMONTE**, S. BERCHI***
}

\begin{abstract}
RÉSUMÉ
Dans le but d'analyser et d'estimer le risque spatial en matière de leishmanioses cutanées et viscérales de l'Est algérien, les auteurs utilisent l'indicateur vectoriel, exprimé en abondances relatives de Phlébotomes. Le découpage bioclimatique préalable permet de définir deux secteurs d'échantillonnage, l'un, septentrional, à climats humide et sub-humide dominants, l'autre méridional, de type aride et peraride. Les prélèvements sont réalisés selon la méthode

des itinéraires-transects. L'analyse des correspondances espèces stations permet d'interpréter les distributions selon le zonage utilisé. La chorologie des différents vecteurs est ainsi précisée : l'un des représentants du sous-genre Larroussius, $P$. perniciosus, responsable de la leishmaniose viscérale en zones sub-humide et semiaride, domine au nord. Phlebotomus papatasi, vecteur de la leishmaniose cutanée zoonotique, est surtout représenté au Sud.
\end{abstract}

\section{Summary: Chorology of Phlebotomus in eastern Algeria. (Diptera, Phlebotomidae).}

To obtain a geographic estimation of the risk of cutaneous and visceral leishmanioses in eastern Algeria, the authors used the vector indicator expressed in relative densities of Phlebotomus species. Two sampling zones were identified, a northen humid-to-semiarid zone and a sourthen arid and perarid zone. Samples were collected according to the itinerary-transect method after bioclimatic stratification. Analysis of correspondences between species and sites was used to interpret distributions according to the zones defined above. The distribution of different vectors was thereby determined: P. (Larroussius) perniciosus, vector of visceral leishmaniasis in the sub-humid and semi-arid bioclimatic zones is dominant in the north. P. (Phlebotomus) papatasi, vector of zoonotic cutaneous leishmaniasis mainly found in the south.
La récente recrudescence des leishmanioses, tant viscérales que cutanées, sur l'ensemble du territoire algérien, a amené les instances décisionnelles de ce Pays, à multiplier les analyses épidémiologiques de foyers. L'étude chorologique des Phlébotomes de l'Est algérien, que nous présentons ici, s'inscrit dans une telle orientation.

\section{MATÉRIELS ET MÉTHODES}

Préalablement à tout échantillonnage, une opération de stratification mésologique a été conduite sur la base de la carte bioclimatique de Cote (2), établie à l'aide des coefficients pluviothermiques d'Emberger (4). Deux secteurs ont été retenus (fig. 1 et 2) : 1) un secteur Nord, de type humide, sub-humide et semi-aride, intéressant l'Atlas tellien (Fédoulès) et les Hauts plateaux cons-

* Laboratoire d'Entomologie fondamentale et appliquée, Campus de Beaulieu, avenue du Général Leclerc, F 35042 Rennes.

** Laboratoire d'Écologie médicale et Pathologie parasitaire, (Pr J.-A. Rioux) Faculté de Médecine, 163 rue AugusteBroussonnet, F 34000 Montpellier.

*** Institut de Biologie, Route d'Aïn el Bey, Constantine, Algérie.

Accepté le : 13 août 1991. tantinois, 2) un secteur Sud, comprenant l'Aurès méridional et le complexe oasien de Biskra, s'étendant sur les étages semi-aride, aride et peraride. Entre ces deux zones, dans la régions d'AïnM'lila, se situait une large bande de terrains salés et de chotts, défavorable aux Phlébotomes. Pour cette raison, les stations correspondantes n'ont pas été prises en compte.

En pratique, les « gîtes de repos » suivants ont été inventoriés : anfractuosités de falaises et d'éboulis, barbacanes de murs, intérieurs de maisons et d'étables. Pour chacune des stations, ont été relevés : la topographie, la végétation, le mésoclimat et le type d'anthropisation (5). La capture proprement dite a été conduite selon la technique des itinéraires-transects à l'aide du piège adhésif simple (6). L'abondance relative de chaque espèce a été exprimée en nombre de Phlébotomes par $\mathrm{m}^{2}$ de piège $\left(\mathrm{P} / \mathrm{m}^{2}\right)$. Au cours de la saison chaude, chaque station a été échantillonnée à l'aide de trente pièges, mis en place pendant une semaine. Sur les 66 stations prises en compte, 34 se situaient dans le secteur septentrional, 32 dans le secteur méridional (fig. 2).

\section{RÉSULTATS}

LISTE DES ESPÈCES

Au total, 6117 Phlébotomes ont été récoltés, se répartissant en 12 espèces (tableau I). L'une d'entre elle, Phlebotomus kazeruni, était nouvelle pour l'Algérie (1). 




\section{Aperçu analytique}

Phlebotomus ariasi Tonnoir, 1921

Répartition géographique : sous-région méditerranéenne occidentale en bioclimats humide et sub-humide. Localités de récolte: Azeba, El Milia. Observé dans le nord de l'Algérie et les Aurès (3).

Phlebotomus longicuspis Nitzulescu, 1930

Répartition géographique : Méditerranée Sud occidentale : Maghreb sub-humide, semi-aride et aride; Espagne. Loca- 
FIG. 2. - Transect de piégeage. Les 66 stations échantillonnées se répartissent en deux secteurs : au nord d'El Milia, sont intéressés les étages bioclimatiques humide et sub-humide. Au sud, (Mâafa, El Kantara et Biskra) se distribuent les étages aride et peraride. L'étage semi-aride est représenté dans les deux secteurs. Dans le secteur sud, l'étage sub-humide des Aurès n’a pas été échantillonné.

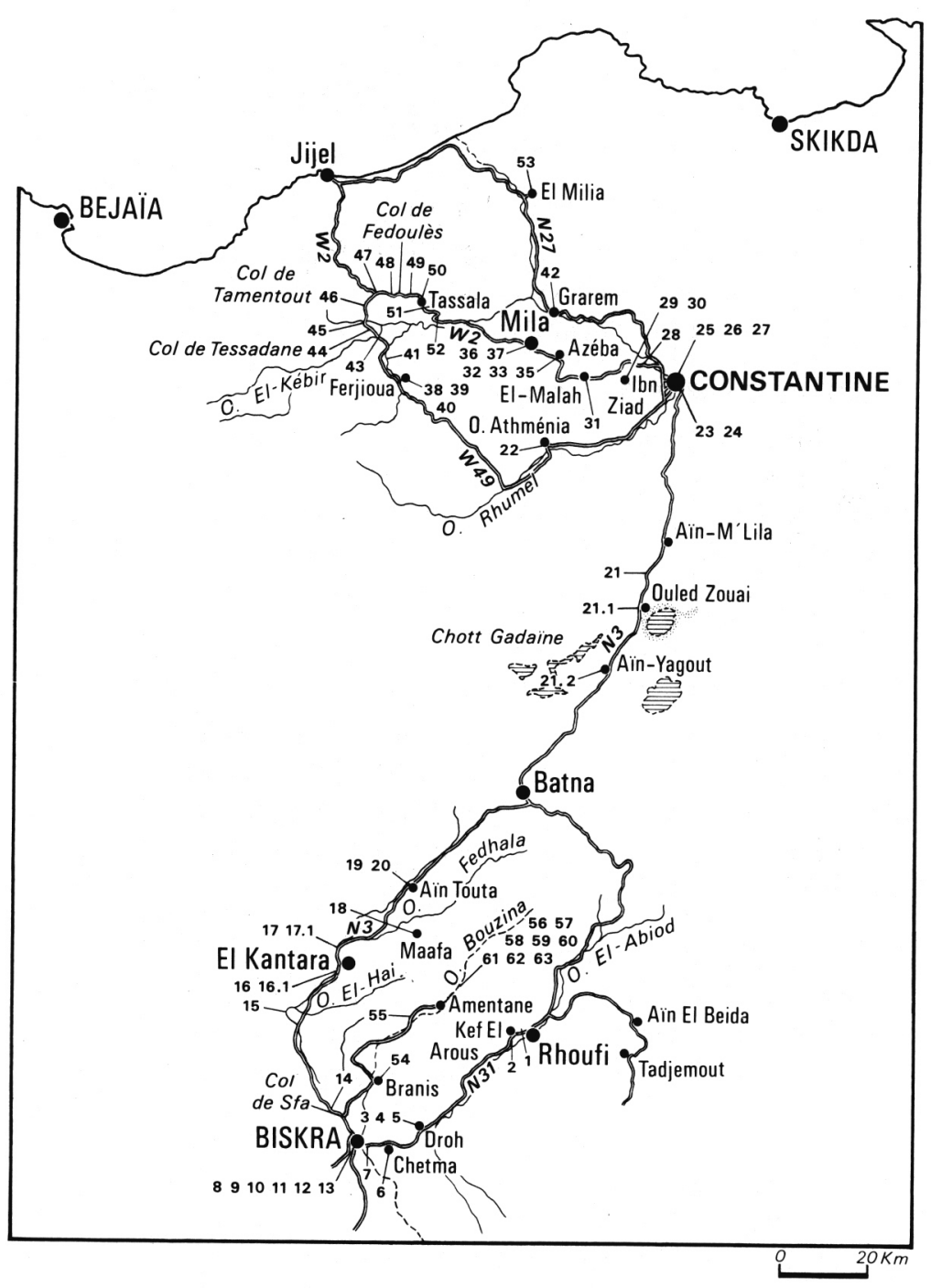

lités de récolte : Amentane, Biskra. Espèce représentée uniquement dans les étages aride et peraride.

\section{Phlebotomus perfiliewi Parrot, 1930}

Répartition géographique : Méditerranée orientale et sudoccidentale. Localités de récolte: Azeba, El-Malah, El Milia, Ferjioua, Grarem, Ibn-Ziad, Tassadane. Au nord d'El-Malah, l'espèce atteint l'étage semi-aride, aussi bien en sites sauvages $\left(2,4 \mathrm{P} / \mathrm{m}^{2}\right)$ qu'urbains $\left(2,1 \mathrm{P} / \mathrm{m}^{2}\right)$.

Phlebotomus perniciosus Newstead, 1911

Répartition géographique : Bassin méditerranéen occidental, de l'humide au semi-aride. Localités de récolte : Aïn-M'lila, Aïn-Yagout, Aïn Touta, Amentane, AzebaBiskra, Constantine, Droh, El Kantara, El-Malah, El Milia, Fédoulès, Ferjioua, Grarem, Ibn-Ziad, Kef el Arous, Mâafa, Mila, Oued Athmenia, Oued El-Hai, Ouled Zouai, Sfa, Tamentout, Tassadane, Tassala. Espèce omniprésente dans le secteur nord, sous bioclimats humide $\left(20,5 \mathrm{P} / \mathrm{m}^{2}\right)$, sub-humide $\left(21,3 \mathrm{P} / \mathrm{m}^{2}\right)$ et semi-aride $\left(34,9 \mathrm{P} / \mathrm{m}^{2}\right)$, tant dans les biotopes urbains (Constantine $79 \mathrm{P} / \mathrm{m}^{2}$ ) que sauvages $\left(44 \mathrm{P} / \mathrm{m}^{2}\right)$. Dans le secteur sud, descend jusqu'à l'étage peraride, dans les rochers et les gabions $\left(10,5 \mathrm{P} / \mathrm{m}^{2}\right)$.

Phlebotomus papatasi (Scopoli, 1786)

Répartition géographique : de l'Inde au Bassin méditerranéen. Localités de récolte : Biskra, Droh, El Kantara, El-Malah, Kef el Arous, Oued Athménia, Tassala. Espèce peu représentée dans notre étude; plus fréquente au Sud (tableau I).

Phlebotomus alexandri Sinton, 1928

Répartition géographique : de la Chine au Maghreb et à l'Espagne. Elément aride à tendance péraride. Localités de récolte : Biskra, Droh, El Kantara, Kef el Arous, Oued El-Hai. 
Phlebotomus kazeruni Theodor et Mesgali, 1964

Répartition géographique : élément irano-touranien et saharo-sindien. Localité de récolte : une seule station de capture, Tassala, à l'étage humide.

Phlebotomus sergenti (Parrot, 1917)

Répartition géographique : de l'Inde et l'URSS à la Méditerranée occidentale. Localités de récolte : Constantine, Tassala. Observé seulement dans le secteur Nord.

Sergentomyia antennata (Newstead, 1912)

Répartition géographique : du Moyen Orient à l'Afrique. Localités de récolte : Aïn M'lila, Aïn Touta, Amentane, Biskra, Chetma, Droh, El Kantara, Kef el Arous, Mâafa, Oued el Hai, Rhoufi, Sfa. Elément érémien, habituellement observé dans les étages aride et peraride; une seule station à l'étage semi-aride.

Sergentomyia fallax (Parrot, 1912)

Répartition géographique : Afrique du Nord. Localités de récolte : Aïn Touta, Amentane, El Malah, Kef el Arous, Mâafa, Oued El-Hai, Sfa, Tassala. Elément présent sur l'ensemble du transect, plus abondant au Sud, sous bioclimats aride et peraride.

Sergentomyia minuta (Rondani, 1843)

Répartition géographique : pourtour méditerranéen. Représentée au Maghreb par la «forme » parroti (Adler et Theodor, 1927), à denture cibariale fournie. Localités de récolte : Aïn M'lila, Aïn Touta, Aïn Yagout, Amentane, Azeba, Biskra, Branis, Chetma, Constantine, Droh, El-Malah, El Milia, Fédoulès, Ferjioua, Grarem, Ibn-Ziad, Kef el Arous, Mâafa, Mila, Oued El-Hai, Rhoufi, Sfa, Tamentout, Tassadane, Tassala. Densité maximale à l'étage semi-aride $\left(68 \mathrm{P} / \mathrm{m}^{2}\right)$, diminuant progressivement aux étages sub-humide $\left(48,3 \mathrm{P} / \mathrm{m}^{2}\right)$ et humide $\left(23,7 \mathrm{P} / \mathrm{m}^{2}\right)$ d'une part, aride $\left(4,3 \mathrm{P} / \mathrm{m}^{2}\right)$ et peraride $\left(3,9 \mathrm{P} / \mathrm{m}^{2}\right)$ d'autre part.

Sergentomyia lewisi (Parrot, 1948)

Répartition géographique : espèce transsaharienne. Localités de récolte : Aïn el Beida, Aïn-M'lila, Aïn Touta, Biskra, Constantine; cette dernière station, en zone semiaride.

\section{SYNTHĖSE ET CONCLUSION}

L'analyse des distributions d'abondance (tableau II), montre une diversité spécifique plus faible dans le Nord où les échantillons sont généralement de type bispécifique : $S$. minuta et $P$. perniciosus (indice de Shannon 0,99 bits en secteur septentionnal, contre 1,9 bits en secteur méridional). L'analyse multidimensionnelle (fig. 3) portant sur la totalité des stations, ne donne pas d'information pertinente sur les préférences édaphiques des différentes espèces.

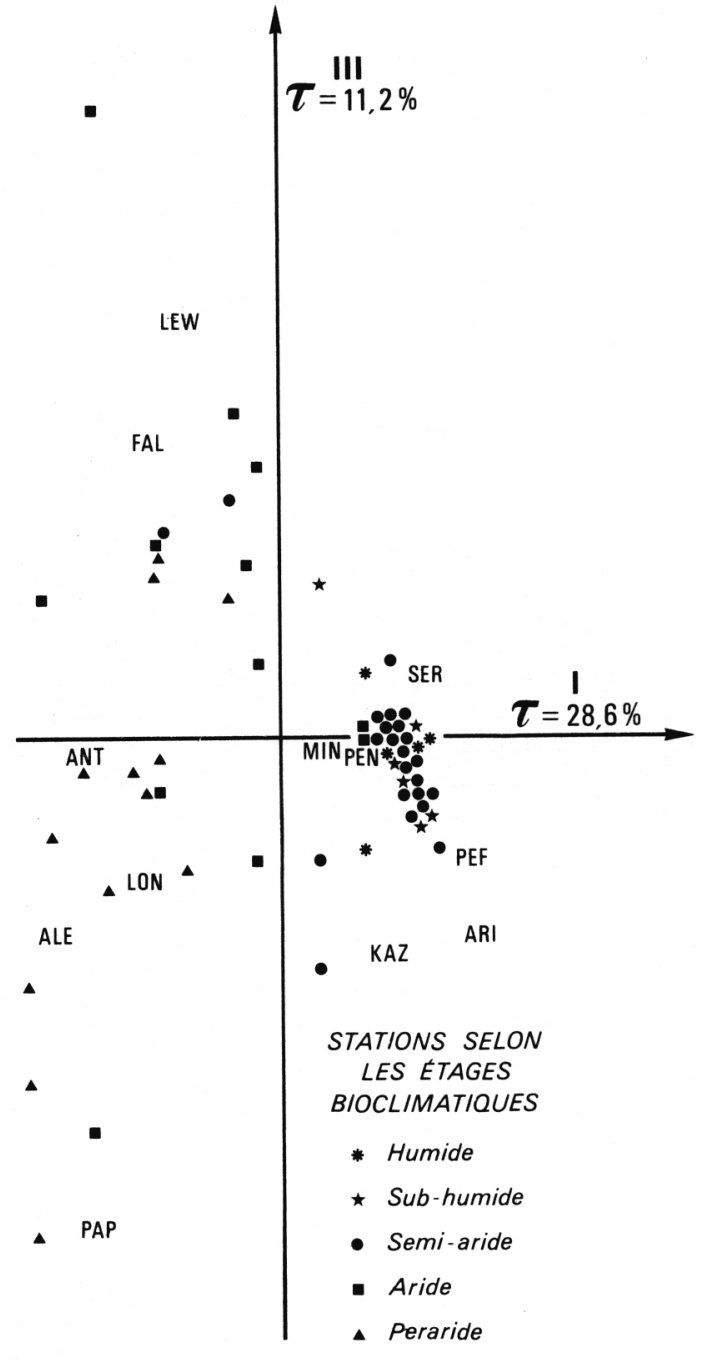

Fig. 3. - Phlébotomes de l'Est algérien. Analyse des correspondances espèces-stations. Le plan I-III regroupe, à droite les stations sous climats humide, sub-humide et semi-aride, à gauche, les stations sous climats aride et peraride. Les espèces sont distribuées de la même façon, en hygromésophiles à gauche, en xérophiles à droite.

ARI P. ariasi; LON P. longicuspis; PER P. perfiliewi; PEN $P$. perniciosus; PAP P. papatasi; ALE P. alexandri; KAZ P. kazeruni; SER P. sergenti; ANT S. antennata; FAL S. fallax; MIN S. minuta; LEW S. lewisi.

Par contre, elle met en évidence un continuum bioclimatique, depuis les espèces érémiennes (S. antennata, $P$. alexandri, $P$. papatasi) jusqu'aux hygrophiles ( $P$. perfiliewi, $P$. ariasi). Une telle distribution a été observée au Maroc (7) à l'occasion d'une étude comparable espècesstations. Dans la présente analyse chorologique des Phlébotomes de l'Est algérien, la répartition des stations correspond à deux aires d'endémie leishmanienne. Au Nord, dans les foyers, connus ou potentiels, à L. infantum et $L$. tropica, dominent $P$. perniciosus et $P$. sergenti. Au Sud, dans la zone à risque pour $L$. major, $P$. papatasi est bien 
TABleAu II. - Fréquence relative des Phlébotomes de l'Est algérien; secteurs nord et sud. Phlebotomus ariasi et $\mathrm{P}$. sergenti sont seulement représentés au Nord. De même P. longicuspis et P. alexandri ne s'observent qu'au Sud. P. perniciosus et S. minuta couvrent les deux secteurs, avec une nette prédominence au Nord.

\begin{tabular}{|c|c|c|c|c|c|c|}
\hline \multirow[b]{2}{*}{ Espèces } & \multicolumn{3}{|c|}{$\begin{array}{c}\text { Secteur nord } \\
\left.\text { (34 stations. } S: 64,32 m^{2}\right)\end{array}$} & \multicolumn{3}{|c|}{$\begin{array}{c}\text { Secteur sud } \\
\left.\text { (32 stations. } S: 28,29 \mathrm{~m}^{2}\right)\end{array}$} \\
\hline & $q+\sigma^{\prime}$ & $P / m^{2}$ & $\%$ & $q+\infty$ & $P / m^{2}$ & $\%$ \\
\hline$P$. ariasi & 2 & 0,1 & 0,1 & & & \\
\hline$P$. longicuspis & & & & 13 & 0,5 & 1,6 \\
\hline P. perfiliewi & 69 & 1,1 & 1,3 & & & \\
\hline P. perniciosus & 1792 & 27,8 & 33,8 & 121 & 4,3 & 14,7 \\
\hline$P$. papatasi & 10 & 0,23 & 0,2 & 15 & 0,5 & 1,8 \\
\hline P. alexandri & & & & 81 & 2,9 & 9,8 \\
\hline P. kazeruni & 1 & 0,0 & 0,0 & . & . & 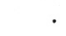 \\
\hline$P$. sergenti & 12 & 0,2 & 0,2 & 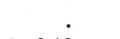 & . & \\
\hline S. antennata & 2 & 0,1 & 0,1 & 342 & 12,1 & 41,6 \\
\hline S. fallax & 6 & 0,1 & 0,1 & 114 & 4,0 & 13,9 \\
\hline S. minuta & 3398 & 52,8 & 64,2 & 128 & 4,5 & 15,3 \\
\hline S. lewisi & 4 & 0,1 & 0,1 & 7 & 0,2 & 0,8 \\
\hline
\end{tabular}

$\mathrm{S}$ : surface $\left(\right.$ en $\left.\mathrm{m}^{2}\right)$ de papier utilisée (recto-verso). $\odot+\mathrm{O}^{+}:$nombre de Phlébotomes. $\mathrm{P} / \mathrm{m}^{2}:$ densité de Phlébotomes par $\mathrm{m}^{2}$ de papier. $\%:$ fréquence relative des espèces.

représenté. Dans l'oasis de Biskra, la présence de $P$. perniciosus, exceptionnelle sous ce climat, expliquerait certains cas sporadiques de leishmaniose viscérale.

Au demeurant, de nombreux problèmes restent encore à résoudre tels que les types de gîtes de repos, les comportements trophiques, la dispersion et la phénologie des espèces vectrices.

Remerciements. - Nos remerciements, vont à la population d'Amentane et plus particulièrement à la famille BouzeKouT ZERARI, pour la cordialité de leur accueil et l'efficacité de leur appui.

\section{RÉFÉRENCES}

1. Berchi S., Rioux J.-A., Belmonte A., Russo J. : Un Phlébotome nouveau pour l'Algérie, Phlebotomus (Paraphlebotomus) kaze- runi. Theodor et Mesghali, 1964. Ann. Parasitol. Hum. Comp., 1986, 61, 507-508.

2. Cote M.: Les régions bioclimatiques de l'est algérien. C.U.R.E.R. éd., Constantine, 1974, 6 p.

3. Dedet J. P., Addadi K. : Épidémiologie des leishmanioses en Algérie. 4. Les phlébotomes (Diptera, Psychodidae) des Aurès. Arch. Inst. Past. Alg., 1977, 52, 85-94.

4. Emberger L. : Une classification biogéographique des climats. Trav. Inst. Bot., Montpellier, 1955, 7, 3-43.

5. Godron M., Daget P., Emberger L., Long G., Le Floc'h, Poissonnet J., Sauvage C., Wacqquant J. P. : Vade Mecum pour le relevé méthodique de la végétation et du milieu. C.N.R.S., Paris, 1969, $169 \mathrm{p}$.

6. Rioux J. A., Golvan Y. J., Croset H., Houin R., Juminer B., Bain O., Tour S. : Écologie des leishmanioses dans le Sud de la France. 1. Les Phlébotomes. Échantillonnage. Éthologie. Ann. Parasitol. Hum. Comp., 1967, 42, 561-603.

7. Rioux J.-A., Rispail P., Lanotte G., Lepart J. : Relations Phlébotomes-bioclimats en écologie des leishmanioses. Corollaires épidémiologiques. L'exemple du Maroc. Bull. Soc. Bot. Fr., 1984, 131, 549-557. 